\title{
Cubes Keep Their Distance
}

\author{
Cubes suspended in a liquid are less likely than spheres to form clusters \\ and fall out of solution.
}

By Rachel Berkowitz

$\square$ article-laden flows are ubiquitous in natural and industrial processes. Models that describe how particles behave in a suspension are vital for, among other things, explaining river deposits and designing waste-water treatment plants. But these models assume that particles are spherical, which is a problem in a world where sediment grains come in all shapes and sizes. Arman Seyed-Ahmadi and Anthony Wachs, of the University of British Columbia, Canada, now show through simulations that, unlike a sphere-laden flow, a cube-laden one tends to resist clustering, remaining homogeneous for longer. The finding provides an important update for models of environmental flows.

Seyed-Ahmadi and Wachs developed numerical simulations that account for the hydrodynamic forces and torques experienced by hundreds of cubic particles suspended in a tank of water. The simulations track the particles' velocity fluctuations, the resulting microstructure of the suspension, and the forces on each particle. The duo found that the spatial distribution of the cubes is more homogenous than a similar mixture of spheres.
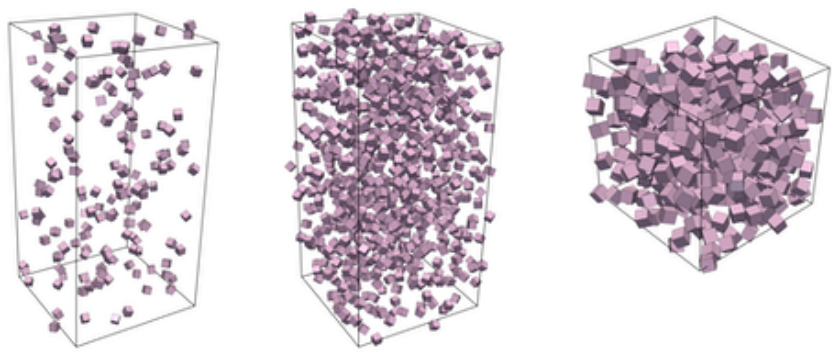

Credit: A. Seyed-Ahmadi and A. Wachs [1]
This result comes from the cubes' sharp edges, which give rise to rotation-induced lift forces that prevent the cubes from clustering. The rotation effectively transfers momentum from the downward direction to the transverse direction, thereby creating a lift-like buoyancy that encourages the particles to remain suspended rather than fall to the bottom.

Seyed-Ahmadi and Wachs conclude that cubic particles may be desirable for industrial applications that need enhanced mixing. And they say the model is a good proxy for other angled grains, such as the particles produced in soil, rock, and mining engineering processes, which are typically polyhedral-shaped.

Rachel Berkowitz is a Corresponding Editor for Physics based in Vancouver, Canada.

\section{REFERENCES}

1. A. Seyed-Ahmadi and A. Wachs, "Sedimentation of inertial monodisperse suspensions of cubes and spheres," Phys. Rev. Fluids 6, 044306 (2021). 